\title{
Prevalência de suicídio e tentativa de suicídio no Brasil
}

\author{
Suicide prevalence and suicide attempt in Brazil
}

\section{Prevalencia de suicidio e intento de suicidio en Brasil}

Kelly Alves Ramos ${ }^{1 *}$, Suzy Tiberly Rodrigues de Souza Araújo ${ }^{1}$, Bárbara Sandra Pinheiro dos Santos $^{2}$, Daiane Carvalho de Sousa ${ }^{2}$, Elielma Ferreira Leite ${ }^{1}$, Geovane Bruno Oliveira Moreira $^{3}$, Helayne Cristina Rodrigues ${ }^{3}$, Pedro Homero Gomes de Sousa ${ }^{3}$, Patrícia Rodrigues da Silva ${ }^{2}$, Ravena de Sousa Alencar Ferreira ${ }^{2}$, Sara Ferreira Coelho ${ }^{3}$, Maira Divina Marques dos Santos ${ }^{2}$, Mayara Kelle Rodrigues de Carvalho ${ }^{2}$, Ysamara Oliveira Vidal ${ }^{4}$.

\section{RESUMO}

Objetivo: Analisar evidências científicas na literatura sobre a prevalência de suicídio e tentativa de suicídio no Brasil no período compreendido entre os anos de 2012 e 2018. Métodos: Trata-se de uma revisão bibliográfica. A busca foi realizada nos bancos de dados SCIELO, MEDLINE e LILACS via Biblioteca Virtual em Saúde (BVS), nos idiomas português, inglês e espanhol, coletados no mês de agosto de 2018. Resultados: A apresentação dos resultados e discussão dos dados obtidos foi realizada de forma descritiva. Para essa apresentação, elaborou-se duas categorias temáticas: Perfil e Fatores Característico do Suicídio e Tentativa de Suicídio. Pôde-se analisar 11 artigos, o perfil das vítimas e os fatores característicos que contribuíram para as ocorrências de tentativa de suicídio e o suicídio. Considerações finais: A tentativa de suicídio ou efetivação do ato não está restrita apenas uma faixa etária ou um público específico, mas consegue atingir adolescentes, adultos e idosos, homens ou mulheres e está relacionada a diversos fatores como depressão, solidão, situação financeira, desesperança e falta de sentido para a vida.

Palavras-chave: Ato, Suicídio, Vítimas.

\section{ABSTRACT}

Objective: To analyze scientific evidence in the literature about the prevalence of suicide and attempted suicide in Brazil between 2012 and 2018. This is an integrative literature review. Methods: It is a literature review. The search was performed in the SCIELO, MEDLINE and LILACS databases via Virtual Health Library (VHL), in portuguese, english and Spanish languages, collected in the month of August 2018. Results: The presentation of the results and discussion of the obtained data was performed descriptively. For this presentation, two thematic categories were elaborated: Profile and Factors Characteristic of Suicide and Attempted Suicide. It was possible to analyze 11 articles, the profile of the victims and the characteristic factors that contributed to the occurrences of suicide attempt and suicide. Final considerations: The attempted suicide or enforcement of the act is not restricted to only one age group or a specific public, but it reaches adolescents, adults and the elderly, men or women and is related to several factors such as depression, hopelessness and lack of meaning for life.

Keywords: Act, Suicide, Victims.

1 UNINOVAFAPI. Teresina, Piauí. *E-mail: kellyalves71@gmail.com

2 Universidade Estadual do Piauí (UESPI). Teresina, Piauí.

3 Universidade Federal do Piauí (UFPI), Teresina, PI.

${ }^{4}$ Faculdade Estácio CEUT, Teresina, PI. 


\section{RESUMEN}

Objetivo: Analizar la evidencia científica en la literatura sobre la prevalencia del suicidio y el intento de suicidio en Brasil entre 2012 y 2018. Métodos: Se trata de una revisión bibliográfica. La búsqueda se realizó en las bases de datos SCIELO, MEDLINE y LILACS a través de la Biblioteca Virtual en Salud (BVS), en los idiomas portugués, inglés y español, recogidos en el mes de agosto de 2018. Resultados: La presentación de los resultados y la discusión de los datos obtenidos se realizó de forma descriptiva. Para esta presentación, se elaboraron dos categorías temáticas: perfil y factores característicos del suicidio y intento de suicidio. Fue posible analizar 11 artículos, el perfil de las víctimas y los factores característicos que contribuyeron a la ocurrencia de intentos de suicidio y suicidio. Consideraciones finales: El intento de suicidio o efectividad del acto no está restringindo a um solo grupo de edad o um público específico, pero logra alcanzar adolescentes, adultos y ancianos, hombres o mujeres y está relacionada con diversos factores como depresión, soledad, situación financeira, desesperanza y falta de sentido para la vida.

Palabras clave: Acto, Suicidio, Víctimas.

\section{INTRODUÇÃO}

O suicídio resulta da interação de fatores de ordem filosófica, antropológica, psicológica, biológica e social e a sua consumação deriva de um ato provocado e realizado pelo indivíduo com o objetivo de pôr fim à vida (RIBEIRO DB, et al., 2016). Assim, é considerado um fenômeno humano complexo, universal e representa um grande problema de saúde pública em todo o mundo. Além de definir-se o suicídio como a morte de uma pessoa causada por autoagressão, esse fenômeno também inclui desde a motivação e ideação de autoextermínio, até o planejamento do ato, a tentativa e o óbito (FÉLIX TA, et al., 2016).

A literatura mostra que a associação entre suicídio e transtornos mentais é de mais de 90\% (BARBOSA FO, et al., 2011). Desse modo, os motivos de um suicídio são invariavelmente mais complexos que um acontecimento recente, como a perda do emprego ou um rompimento amoroso. A existência de um distúrbio mental encontra-se presente na maioria dos casos. Dentre eles, os mais comumente associados são: depressão, transtorno do humor bipolar e dependência de álcool e de outras drogas psicoativas. A esquizofrenia e algumas características relacionadas à personalidade também são importantes fatores de risco (BOTEGA NJ, 2014).

Quanto aos dados, um milhão de pessoas se suicida no mundo anualmente e a cada 45 segundos, uma pessoa se suicida em algum lugar do planeta. Os países da Europa oriental, américa central e américa do sul apresentam os índices mais altos de suicídio (ASSUMPÇÃO GLS, et al., 2018). A Organização Mundial da Saúde (OMS), em seu primeiro relatório sobre prevenção do suicídio, aponta o Brasil como oitavo país com maior índice de suicídio. Contudo, ressalva que ele é passível de prevenção e destaca ainda a importância de estratégias de trabalho com uma abordagem multisetorial abrangente (MÜLLER AS, et al., 2017).

Neste contexto, o registro epidemiológico dos casos de suicídio e das tentativas em todo o território nacional é fundamental, uma vez que, o conhecimento destas informações serve de embasamento para o planejamento de ações preventivas. Assim, torna-se necessário que diferentes estratégias de prevenção sejam propostas para cada grupo específico (jovens, homens, mulheres, idosos), a fim de evitar mais casos de suicídio na população (MAGALHÃES APN, et al., 2014).

Alguns fatores se transformam em dificuldades para o tratamento daqueles que apresentam transtornos mentais, como o acesso às informações sobre saúde mental, o alto custo dos medicamentos e das terapias, os tabus sociais que rondam a temática, falhas na formação dos médicos quanto à saúde mental, além da falta de especialistas em saúde mental. Assim, vale destacar que a prevenção do suicídio é de suma importância no enfrentamento desta problemática na sociedade moderna (BARBOSA FO, et al., 2011).

Desse modo, considerando o suicídio como um problema de saúde pública, este estudo é relevante, uma vez que o conhecimento do contexto desta problemática torna-se essencial para a sua compreensão, o que 
favorece a construção de estratégias de prevenção e enfrentamento, além de fomentar discussões científicas na área de saúde mental. Este estudo tem como objetivo analisar evidências científicas na literatura sobre o suicídio e tentativa de suicídio no Brasil no período compreendido entre os anos de 2012 e 2018.

\section{MÉTODOS}

Este estudo trata-se de uma revisão bibliográfica, que se caracteriza por agrupar, analisar e sintetizar resultados de pesquisas sobre um determinado tema ou questão, de maneira sistemática e ordenada, a fim de apresentar, discutir e aprofundar conhecimentos acerca da temática proposta (MENDES KDS, et al., 2008).

Os artigos foram selecionados de acordo com os descritores: ato, suicídio e vítima. Os critérios de inclusão foram: artigos indexados nas bases de dados Literatura cientifica e técnica da América Latina e Caribe (LILACS), Sistema Online de Busca e Análise de Literatura Médica (MEDLINE) e Biblioteca Eletrônica Científica Online (SCIELO) via Biblioteca Virtual em Saúde (BVS), disponíveis eletronicamente, no idioma português, inglês e espanhol no período de 2012 a 2018. Com a finalidade de reduzir possíveis vieses nos resultados da pesquisa, foram excluídos artigos de estudo de caso, artigos de opinião, dissertações, teses, livros, capítulos de livros, anais de congresso ou conferências, assim como artigos do tipo teóricos-reflexivos, por não retratarem resultados de investigações.

Analisando todas as bases de dados, foram encontrados 500 artigos aproximadamente. Após a leitura dos títulos dos artigos, notou-se que alguns deles se repetiram nas diferentes bases e outros não preenchiam os critérios deste estudo.

Foram selecionados 100 artigos para a leitura dos resumos e excluídos os que não diziam respeito ao propósito deste estudo. Após a leitura dos resumos, foram selecionados 11 artigos entre os anos de 2012 e 2018 que preenchiam os critérios inicialmente propostos e lidos na íntegra. A coleta de dados foi realizada no mês de agosto de 2018. E de forma sintetizada, elaborou-se duas categorias temática, devido a importância do tema abordado, onde a primeira proposta foi a análise do perfil das vítimas e a segunda a Identificação dos fatores característico no Suicídio e Tentativa de Suicídio.

\section{RESULTADOS E DISCUSSÃO}

A apresentação dos resultados e discussão dos dados obtidos foi realizada de forma descritiva com análise detalhada de cada periódico. No intervalo analisado, o ano de 2018 predominou com seis publicações, 2017 com quatro e 2015 com uma publicação.

Em relação à base de dados, MEDLINE foi a que mais indexou artigos nessa temática, com um total de sete. Enquanto as bases de dados LILACS e SCIELO apresentaram dois artigos cada.

No que diz respeito às abordagens metodológicas, prevaleceu a quantitativa, qualitativa e epidemiológica com duas produções, as demais foram abordagem transversal, pesquisa-intervenção, estudo de caso social, estudo ecológico e retrospectiva com uma produção.

Em relação à região dos estudos, dos onze artigos analisados, quatro autores realizaram sua pesquisa na região Sudeste, três no Nordeste, dois no Sul, um no Norte e um estudou todas as regiões brasileiras.

Para melhor compreensão da análise dos resultados, foi elaborado um quadro em que constam: autor(es)/ano, objetivo do estudo, tipo de estudo/ pacientes e resultados evidenciados (Quadro 1).

Os estudos analisados sobre tentativa de suicídio e suicídio não possuem resultados fidedignos devido a existência de inúmeros casos não notificados ou que previamente não foram identificados. Em uma visão sintética dentro dos artigos selecionados buscou criar duas categorias importantes, onde uma objetivou analisar o perfil das vítimas, e posteriormente, a outra identificar os principais fatores característicos que contribuíram na tentativa de suicídio e no suicídio. 
Quadro 1 - Caracterização dos estudos científicos de acordo com os artigos analisados, Teresina, PI, 2012 a 2018.

\begin{tabular}{|c|c|c|c|}
\hline Autor(es)/Ano & Objetivo do estudo & Tipo de estudo/ Pacientes & Resultados evidenciados \\
\hline Akca SO et al. 2018 & $\begin{array}{l}\text { Determinar níveis psicológicos de } \\
\text { sintomas e probabilidade de } \\
\text { suicídio em jovens. }\end{array}$ & $\begin{array}{l}\text { Estudo transversal. } \\
\text { Pacientes: Jovens entre } 15 \text { e } 24 \\
\text { anos. }\end{array}$ & $\begin{array}{l}\text { O risco de suicídio é maior em jovens } \\
\text { com depressão, ansiedade, } \\
\text { autopercepção negativa e sentimento de } \\
\text { hostilidade. }\end{array}$ \\
\hline Cescon LF et al. 2018 & $\begin{array}{l}\text { Investigar a atenção ao suicídio de } \\
\text { um serviço de atenção psicossocial } \\
\text { em um município de São Paulo. }\end{array}$ & $\begin{array}{l}\text { Pesquisa-intervenção de perspectiva } \\
\text { cartográfica. }\end{array}$ & $\begin{array}{l}\text { As análises revelaram um processo de } \\
\text { trabalho centrado em consultas } \\
\text { psiquiátricas e na medicalização do } \\
\text { sofrimento, no qual a atenção ao suicídio } \\
\text { era pouco problematizada. }\end{array}$ \\
\hline Claumann GS, et al. 2018 & $\begin{array}{l}\text { Estimar a prevalência de pensamentos } \\
\text { e comportamentos suicidas e a } \\
\text { associação com a insatisfação corporal } \\
\text { em } \\
\text { adolescentes. }\end{array}$ & $\begin{array}{l}\text { Estudo epidemiológico } \\
\text { Transversal, de base escolar. } \\
\text { Participantes: Adolescentes }\end{array}$ & $\begin{array}{l}\text { O sexo feminino apresentou } \\
\text { maiores prevalências de pensamento, } \\
\text { planejamento e tentativa de suicídio } \\
\text { comparado ao masculino. }\end{array}$ \\
\hline Ribeiro DB et al. 2018 & $\begin{array}{l}\text { Analisar o perfil epidemiológico dos } \\
\text { casos de tentativas de suicídios e } \\
\text { suicídios nos bancos de dados do } \\
\text { Sistema de Informação de } \\
\text { Mortalidade e Sistema de Informação } \\
\text { de Agravos de Notificação e a } \\
\text { tendência temporal da mortalidade } \\
\text { por suicídio. }\end{array}$ & $\begin{array}{l}\text { Estudo quantitativo e de série } \\
\text { temporal, utilizou-se as bases de } \\
\text { dados do Sistema de Informação de } \\
\text { Mortalidade para os óbitos por } \\
\text { suicídio e o Sistema de Informação } \\
\text { de Agravos de Notificação. }\end{array}$ & $\begin{array}{l}169 \text { tentativas de suicídio e } 11 \text { óbitos por } \\
\text { suicídios foram analisados. A tentativa } \\
\text { de suicídio é predominante em } \\
\text { adolescentes e adultos jovens, mulheres } \\
\text { tentam mais suicídio do que os homens } \\
\text { através de métodos menos agressivos. } \\
\text { A mortalidade é maior entre } 30 \text { e } 49 \\
\text { anos, } 45,4 \% \text { por enforcamento. }\end{array}$ \\
\hline Silva ES et al. 2018 & $\begin{array}{l}\text { Analisar a sua ocorrência e caracterizar } \\
\text { os casos de suicídio registrados em um } \\
\text { município da Amazônia Legal, entre os } \\
\text { anos de } 1999 \text { e } 2016 \text {. }\end{array}$ & $\begin{array}{l}\text { Estudo epidemiológico descritivo e } \\
\text { transversal. Casos registrados como } \\
\text { suicídios no Instituto Médico Legal } \\
\text { (IML). }\end{array}$ & $\begin{array}{l}\text { Conclui-se que o gênero masculino e a } \\
\text { faixa etária adultos foram mais } \\
\text { propensos a cometer a autoagressão. }\end{array}$ \\
\hline Botti NCL et al. 2018 & $\begin{array}{l}\text { Identificar características } \\
\text { comportamento suicida e fatores de } \\
\text { risco de homens e mulheres com } \\
\text { transtornos } \\
\text { psiquiátricos. }\end{array}$ & $\begin{array}{l}\text { Estudo quantitativo } \\
\text { de } 410 \text { prontuários de pacientes } \\
\text { psiquiátrico em tratamento. }\end{array}$ & $\begin{array}{l}\text { Evidenciaram-se particularidade do } \\
\text { comportamento suicida entre pacientes } \\
\text { psiquiátricos em tratamento em relação } \\
\text { ao sexo. }\end{array}$ \\
\hline
\end{tabular}

$\overline{\text { REAS/EJCH | Vol.Sup.32 | e1244 | DOI: https://doi.org/10.25248/reas.e1244.2019 Página } 4 \text { de } 7}$ 


\begin{tabular}{|c|c|c|c|}
\hline Minayo MCS et, al. 2017 & $\begin{array}{l}\text { Analisadas } 16 \text { histórias de vida de } \\
\text { homens e mulheres idosos que em nove } \\
\text { Instituições de Longa Permanência no } \\
\text { Estado do Rio de Janeiro e que tentaram } \\
\text { dar cabo à vida ou têm comportamento } \\
\text { suicida }\end{array}$ & $\begin{array}{l}\text { Estudo qualitativo } \\
\text { foi precedido por uma pesquisa do } \\
\text { perfil de } 122 \text { idosos }\end{array}$ & $\begin{array}{l}\text { Os principais fatores comuns a homens } \\
\text { e mulheres institucionalizados em } \\
\text { situação de risco para a morte }\end{array}$ \\
\hline Meneghel SN e Moura R (2017) & $\begin{array}{l}\text { Reflexão sobre a cultura alemã e a } \\
\text { ética do trabalho como determinantes } \\
\text { do suicídio, em um município } \\
\text { colonizado por alemães e cuja } \\
\text { atividade predominante está ligada à } \\
\text { monocultura do fumo. }\end{array}$ & $\begin{array}{l}\text { Estudo de caso social, } 14 \\
\text { informantes chave. }\end{array}$ & $\begin{array}{l}\text { As reflexões dos entrevistados apontam } \\
\text { para o suicídio como um recurso usado } \\
\text { na cultura desses descendentes de } \\
\text { alemães para enfrentar as dificuldades. }\end{array}$ \\
\hline Santos EGO e Barbosa IR (2017) & $\begin{array}{l}\text { Avaliar a distribuição espacial da } \\
\text { mortalidade por suicídios no Nordeste } \\
\text { do Brasil e sua relação com indicadores } \\
\text { sociais e econômicos, buscando } \\
\text { identificar o padrão dessa distribuição } \\
\text { por meio de } \\
\text { uma análise go-estatística. }\end{array}$ & $\begin{array}{l}\text { Estudo Ecológico que } \\
\text { utilizou dados de óbitos por suicídio } \\
\text { registrados no SIM. Aplicados os testes } \\
\text { Moran Global e Local e a análise } \\
\text { bivariada LISA. }\end{array}$ & $\begin{array}{l}\text { Conclui-se que a mortalidade por } \\
\text { suicídio no Nordeste do Brasil } \\
\text { apresenta distribuição aleatória e não } \\
\text { guarda relação espacial com variáveis } \\
\text { socioeconômicas. }\end{array}$ \\
\hline Leite SCA, et al. (2017) & $\begin{array}{l}\text { Caracterizar o perfil epidemiológico de } \\
\text { casos de suicídios na cidade de Piripiri- } \\
\text { PI nos anos de } 2008 \text { a } 2012 \text {. }\end{array}$ & $\begin{array}{l}\text { Análise retrospectiva, quantitativa, } \\
\text { perfil e exploratória, foram utilizadas } \\
\text { variáveis disponíveis no SIM- } \\
\text { DATASUS. }\end{array}$ & $\begin{array}{l}\text { O número de mortes foram de } 35 \text {, o } \\
\text { sexo predominante foi o masculino, } \\
\text { sendo o método mais utilizado o de } \\
\text { enforcamento; a cor predominante foi a } \\
\text { parda e o local escolhido para } \\
\text { consumação foi o domicílio. }\end{array}$ \\
\hline Gonçalves PIE, et al. (2015) & $\begin{array}{l}\text { Investigar as percepções de } \\
\text { profissionais que atuam na } \\
\text { emergência do Hospital Regional do } \\
\text { Agreste Pernambucano sobre } \\
\text { comportamento suicida a relação } \\
\text { destas com as práticas } \\
\text { de cuidado. }\end{array}$ & $\begin{array}{l}\text { Pesquisa qualitativa, realizada } \\
\text { observação com registro em diário de } \\
\text { campo e entrevistas semiestruturadas. } \\
\text { Participantes: nove profissionais de } \\
\text { saúde. }\end{array}$ & $\begin{array}{l}\text { O estudo ressalta a importância de } \\
\text { ações de educação permanente na } \\
\text { unidade hospitalar que contribuam para } \\
\text { a compreensão dos fatores } \\
\text { relacionados ao comportamento suicida } \\
\text { e as práticas de cuidado. }\end{array}$ \\
\hline
\end{tabular}

Fonte: Ramos KAR, Araújo STRS, Santos BSP, et al., 2018. 


\section{Perfil das vítimas da tentativa suicídio e suicídio}

De acordo com análise dos artigos selecionados, as vítimas que se propõem a tentativa de suicídio ou até chegar as vias de fatos, que é a conclusão do ato do suicídio, não possuem uma padronização. Inicialmente, identifica os jovens/adolescentes em uma faixa etária de 15 -24, estudantes, maioria sexo feminino, que estão enfrentando vários desafios e responsabilidades constantes impostas pela sociedade e por familiares (AKCA SO et al. 2018; CLAUMANN GS et al. 2018; RIBEIRO DB et al. 2018).

Quando se busca investigar a população adulta, percebe que homens e mulheres são acometidos em proporções equivalentes, mas cada um suas particularidades, em uma faixa etária de 30-45 anos, geralmente são solteiros e desempregados (BOTTI NCL et al. 2018; LEITE SCA, et al. 2017; SANTOS EGO e BARBOSA IR, 2017).

Os idosos são incluídos também nas ocorrências de casos notificados e não possuem uma faixa etária especifica nos artigos abordados, mas são analisados na categoria seguinte, quanto aos fatores preexistentes que antecipam a tentativa do suicídio e o suicídio (MINAYO MCS et al. 2017).

\section{Os principais fatores característicos que contribuíram na tentativa de suicídio e no suicídio}

De acordo com os achados do quadro 1, os principais fatores que contribui na tentativa de suicido e no suicídio são: problemas psicológicos como depressão, ansiedade, autopercepção negativa e sentimento de hostilidade, comportamentos suicidas e a associação com a insatisfação corporal entre adolescentes, problemas socioeconômicos, exclusão social devidos características raciais e sexuais, abandono familiar principalmente entre os idosos devido incapacidade e dependência dos mesmos. Ainda pode identificar que os homens estão mais propensos a cometerem a autoagressão, geralmente por enforcamento e com efetivação em domicílio (AKCA SO et al. 2018; MENEGHEL SN e MOURA R, 2017; RIBEIRO NM, et al. 2018)

Os profissionais de saúde são habilitados para acolher e identificar esses pacientes, objetivando a prevenção, tratamento e reabilitação incluindo programas de promoção da saúde, com abordagem e acompanhamentos buscando a preservação da vida. Estudos analisam a importância de ações de educação permanente no âmbito profissional, que buscam contribuir na compreensão dos fatores relacionados ao comportamento suicida e as práticas de cuidado, onde a problematização da tentativa de suicídio e suicídio é abordada como um problema de saúde pública (GONÇALVES PIE, et al. 2015; CESCON LF et al. 2018).

\section{CONSIDERAÇÕES FINAIS}

A partir da análise das produções científicas, foi possível verificar que, quanto ao perfil e fatores característicos, observou-se que a tentativa de suicídio ou efetivação do ato não está restrita a apenas uma faixa etária ou público específico, mas a sociedade como um todo atingindo adolescentes, adultos, idosos, homens e mulheres. Identificou-se também a relação deste problema com diversos fatores como depressão, solidão, situação financeira, desesperança, falta de sentido para a vida, entre outros. Além disso, destacouse a possibilidade de intervenção com a realização do diagnóstico e tratamento, abordado por profissionais de saúde capacitados para acolher e identificar fatores característicos que podem resultar na tentativa do suicídio e no suicídio.

\section{REFERÊNCIAS}

1. AKCA SO, et al. Mental status and suicide probability of young people: A cross- sectional study. Revista da Associação Médica Brasileira, 2018; 64 (1): 32-40.

2. ASSUMPÇÃO GLS, et al. Depressão e suicídio: uma correlação. Pretextos- Revista da Graduação em Psicologia da PUC Minas, 2018; 3 (5): 312-333.

3. BARBOSA FO, et al. Depressão e o suicídio. Rev. SBPH, $2011 ; 14$ (1): 233-243.

4. BOTEGA NJ. Comportamento suicida: epidemiologia. Psicologia USP, 2014; 25 (3): 231-236

5. BOTTI NCL, et al. Características e fatores de risco do comportamento suicida entre e mulheres com transtornos psiquiátricos. Cogitare Enfermagem, 2018; 23 (2): 1-10. 
6. CESCON LF, et al. Aproximações e distanciamentos ao suicídio: analisadores de um serviço de atenção psicossocial. Saúde Social, 2018; 27 (1): 185-200.

7. CLAUMANN GS, et al. Prevalência de pensamentos e comportamentos suicidas e associação com a insatisfação corporal em adolescentes. Jornal Brasileiro de Psiquiatria, 2018; 67 (1): 3-9.

8. FÉLIX TA, et al. Fatores de risco para tentativa de suicídio: produção de conhecimento no Brasil. Revista Contexto \& Saúde, 2016; 16 (31): 173-185.

9. GONÇALVES PIE, et al. Comportamento Suicida: Percepções e Práticas de Cuidado? Psicologia Hospital (São Paulo), 2015; 13 (2): 64-87.

10. LEITE SCA, et al. Caracterização do perfil epidemiológico de casos de suicídios na cidade de Piripiri-PI nos anos de 2008 a 2012. Revista Interdisciplinar, 2017; 10 (1): 103-109.

11. MAGALHÃES APN, et al. Atendimento a tentativas de suicídio por serviço de atenção pré-hospitalar. Jornal Brasileiro de Psiquiatria, 2014; 63 (1): 16-22.

12. MENDES KDS, et al. Revisão Integrativa: Método da Pesquisa Para a Incorporação de Evidências na Saúde e na Enfermagem. Texto contexto - Enfermagem, 2008; 17(4): 758-764.

13. MENEGHEL SN, MOURA R. Suicide, culture and work in a German-colonized municipality in the South of Brazil. Interface (Botucatu), 2017; 22 (67): 1135-1146.

14. MINAYO MCS, et al. O comportamento suicida de idosos institucionalizados: histórias de vida. Physis: Revista de Saúde Coletiva, 2017; 27 (4): 981-1002.

15. MÜLLER AS, et al. Estratégias de prevenção e pósvenção do suicídio: Estudo com profissionais de um Centro de Atenção Psicossocial. Revista de Psicologia, IMED, 2017; 9 (2): 6-23.

16. RIBEIRO DB, et al. Motivos da tentativa de suicídio expressos por homens usuários de álcool e outras drogas. Revista Gaúcha Enfermagem, 2016; 37 (1): 1-7.

17. RIBEIRO NM, et al. Análise da tendência temporal do suicídio e de Sistemas de Informações em Saúde em relação às tentativas de suicídio. Texto contexto- enfermagem, 2018; 27 (2): 1-11.

18. SANTOS EGO, BARBOSA IR. Conglomerados espaciais da mortalidade por suicídio no nordeste do Brasil e sua relação com indicadores socioeconômicos. Caderno Saúde Coletiva, 2017; 25 (3): 371-378.

19. SILVA ES, et al. Perfil de suicídios em município da Amazônia Legal. Caderno Saúde Coletiva, 2018 ; 26 (1): $84-91$. 